\title{
LA INTENCIÓN EMPRENDEDORA UNIVERSITARIA: \\ UNA PERSPECTIVA DE GÉNERO EN QUERÉTARO
}

\section{THE UNIVERSITY ENTREPRENEURIAL INTENTION: \\ A GENDER PERSPECTIVE IN QUERETARO}

\author{
Flor Brown-Grossman \\ (iD) orcid.org/0000-0001-9943-6741 \\ Universidad Autónoma de Querétaro \\ México \\ brown@unam.mx
}

\section{Michael Demmler}

(iD) orcid.org/0000-0002-1629-5814

Universidad Autónoma de Querétaro

México

michael.demmler01@gmail.com

\section{Selene María López Muñoz}

Universidad Autónoma de Querétaro

México

slopez25@alumnos.uaq.mx

\begin{abstract}
The present study analyses different factors affecting entrepreneurial intention of men and women. Based on a survey applied to students of the Autonomous University of Queretaro, we used demographic characteristics of students and factor analysis to estimate a Logit model. The results show that women are less likely to undertake entrepreneurial projects compared to men. Other important differences were observed in the variables "education level", "personal entrepeneurial characteristics", "presence of entrepreneurs in the family" and "access to funding". The findings show the necessity to design public policies that promote entrepreneurship in women.
\end{abstract}

Keywords: Entrepreneurial Intention, Higher Education, gender, Mexico.

\begin{abstract}
Resumen
Se analizaron los diferentes factores que inciden en la intención emprendedora de hombres y mujeres. A partir de una encuesta aplicada a alumnos de la Universidad Autónoma de Querétaro, usamos características demográficas de los alumnos y análisis factorial para estimar un modelo Logit. Los resultados mostraron que las mujeres tienen menos probabilidades de emprender que los hombres. Otras divergencias fueron el "nivel de la carrera", "características personales empresariales", "presencia de empresarios en la familia" y "acceso al financiamiento". Estos hallazgos muestran la necesidad de diseñar políticas públicas que fomenten el emprendimiento en mujeres.
\end{abstract}

Palabras clave: Intención Emprendedora, Educación Superior, género, México. 


\section{Introducción}

El estudio del emprendimiento ha llamado la atención de académicos, instituciones y empresarios debido a la contribución en la generación de empleos, las innovaciones y su participación en el desarrollo tanto económico como social (Urbano y Toledano, 2008). El emprendimiento hoy día es un impulsor importante de la economía y, por ende, su estudio ha cobrado relevancia en la comunidad científica. Dentro del marco de las políticas públicas, surge la necesidad de evitar la discriminación, exclusión y desigualdad de mujeres y hombres para crear condiciones que permitan cerrar la brecha de género en cuanto al emprendimiento.

Recientemente se reconoce el hecho de que las mujeres tienen un papel sobresaliente en el crecimiento de la economía. Si la disparidad de género se acortara en México, para 2025 se incorporarían casi 800 mil miIlones de dólares al Producto Interno Bruto (PIB) y 12 billones de dólares al PIB mundial (Bolio et al., 2018). Sin embargo, la participación femenina en el emprendimiento enfrenta "problemas específicos, dificultades y obstáculos", como la falta de acceso a los recursos financieros, capital y barreras culturales, políticas y jurídicas (Quintos, 2019; The World Bank, s.f.).

Estas condiciones crean brechas de género en la actividad emprendedora. En México, la Tasa de Actividad Emprendedora (TEA) es mayor para hombres que para mujeres $(13.60 \%$ y $12.41 \%$, respectivamente); para Querétaro la diferencia es mayor, ya que la TEA de los hombres es de $16.98 \%$, mientras que la de las mujeres es de $8.63 \%$. En cuanto a la percepción de sus capacidades para emprender, la mujer queretana está por encima de la nacional (71.62\% contra $70.68 \%$ ). En los conocimientos, habilidades y experiencias, los hombres parecen tener una mejor percepción de sí mismos en comparación con las mujeres (76.15\% contra $67.62 \%$, respectivamente). Por último, la Intención Emprendedora en Querétaro resulta menor (20.86\%) al promedio nacional (23.00\%). Al tomar en cuenta esta intención, los hombres mostraron que sus expectativas de iniciar un negocio son mayores al promedio nacional (29.06\%); por el contrario, las expectativas de las mujeres son menores (13.65\%) (Alonso Galicia et al., 2019).

Esta investigación pretende explicar el origen de esta situación al identificar los factores que se encuentran al momento de emprender en las decisiones de hombres y mujeres, como son las aptitudes, intenciones, roles, características e ideas. A la vez, el objetivo es evaluar si alguno de estos factores tiene más peso que otro dependiendo del género. Así, la investigación intenta contestar la siguiente pregunta: ¿Cuáles son los factores que se encuentran en la intención de emprender en mujeres y hombres? Se pretende mostrar que existen diferencias en la probabilidad de emprender entre hombres y mujeres y que los factores que inciden en su emprendimiento no son iguales.

Este trabajo, además de la presente introducción, cuenta con otras tres secciones. Subsecuentemente se muestra la revisión de la literatura en cuanto al emprendedor y los factores determinantes del emprendimiento, modelos de intención y un panorama del género en el emprendimiento. Posteriormente se expone la metodología usada para analizar los factores de la intención emprendedora entre los hombres y mujeres: diseño del cuestionario, análisis factorial y modelo Logit. Posteriormente se exhiben el análisis y la discusión de los resultados obtenidos. Las conclusiones y reflexiones se encuentran en la última sección.

\section{Revisión de la literatura Emprendimiento, emprendedurista y sus características}

Para observar los factores que se encuentran en una persona al momento de emprender, es necesario conocer la definición de los conceptos emprendimiento y emprendedurista, que han sido analizados desde las perspectivas psicológica, económica, sociológica y antropológica (Philipsen, 2011); de ahí la diversidad de definiciones como se observa en los siguientes ejemplos. El em- 
prendedurista es un sujeto - por lo que no es un colectivo ni una institución-, es un actor económico y es creador de una nueva institución jurídicamente definida como empresa (Pfeilstetter, 2011). Es el individuo que percibe una idea y la lleva a cabo a través de la administración, la innovación y la toma de riesgos (Kets de Vries, 1977). El emprendedurista observa una oportunidad y desarrolla una empresa para explotarla (Pereira Laverde, 2003).

Para McClelland, tiene características como el ser original, responsable, innovador, cuenta con una ligera aversión al riesgo, conoce los resultados que dieron sus acciones y planifica tomando en cuenta el largo plazo (citado en Arboleda, 2014).

No es la intención realizar una revisión exhaustiva de estas definiciones; en nuestra opinión, y para los fines de esta investigación, consideramos al emprendedurista como el agente económico que es autoconfiado, observa y evalúa nuevas oportunidades con la que hará negocios y contribuirá al bienestar económico de su país.

Los factores influyentes del emprendimiento también han sido estudiados y agrupados desde distintos puntos de vista por los autores.

Por ejemplo, Ruiz Navarro et al. (2012) y Arenius y Minniti (2005) al analizar la actividad emprendedora femenina en relación con la masculina, los agruparon en demográficos (edad, educación, riqueza, situación laboral y capital humano), y de percepción (autoconfianza, reconocimiento de oportunidades, habilidades, fracaso, conocimiento de otros empresarios y tolerancia al riesgo). Por su parte, Jiménez-Cercado et al. (2017) los clasifican en internos (conocimiento, factibilidad percibida, actitud, normas sociales) y externos (características demográficas o de fondo).

Recientemente ha cobrado importancia el analizar los factores internos y externos que se encuentran a la hora de emprender; los modelos de intención permiten examinar estos aspectos. Dada su relevancia, se expone brevemente a continuación.

\section{Los modelos de Intención Emprendedora}

En la presente sección se exhiben algunas características de los modelos de intención, que son un buen arranque para el análisis del emprendimiento. La intención es un requerimiento imprescindible tanto para ser emprendedurista como para llevar a cabo comportamientos específicos después de la fase inicial (Liñán, 2004). El emprendimiento no se inicia con un reflejo, sino que hay una intención detrás de la acción de emprender; se procesan las señales del ambiente en el que se encuentra la persona para empezar a desarrollar la oportunidad en una idea comercializable. La intención de realizar un comportamiento obedece a las actitudes que el individuo tiene hacia dicho comportamiento (Ajzen, 1991). Las intenciones dependen de las actitudes que son la medida en que la persona evalúa positiva o negativamente alguna situación (Liñán, 2004). A su vez, las actitudes se ven afectadas por factores exógenos. Cuanto mayor intención se encuentra en el individuo, la probabilidad de que se involucre en la conducta será más grande. Por tanto, la Intención Emprendedora es considerada como un buen predictor del futuro involucramiento para la realización de nuevos negocios (Shapero y Sokol, 1982; Ajzen, 1991; Krueger y Carsrud, 1993).

Existen modelos de intención que se utilizan para entender la conducta emprendedurista, algunos de ellos son:

1. El Modelo del Evento Emprendedor (MEE) de Shapero y Sokol (1982) prioriza el proceso emprendedurista. Las intenciones del emprendedurista dependen de la deseabilidad personal, factibilidad y propensión al actuar.

2. Modelo de la Teoría del Comportamiento Planeado (MTCP) de Ajzen (1991), el cual señala que las intenciones del individuo dependerán de las percepciones personales, las normas sociales y la viabilidad.

El modelo más utilizado es el de Ajzen (1991). El autor explica que la intención de un individuo depende de sus creencias debido a que las normas sociales son el antecedente inmediato de la conducta. De 
acuerdo con el autor, son tres los factores que inciden en la intención: 1) La actitud hacia la conducta (grado en que los individuos perciben el atractivo del comportamiento en cuestión), 2) Las normas que son subjetivas (presión social percibida para realizar el comportamiento en cuestión), y 3) El control que se percibe sobre dicha conducta (autoevaluación de la propia competencia con respecto a la tarea o comportamiento).

Lu y Wang (2018) encontraron que existe un vínculo entre el Comportamiento Planeado sobre la Intención Emprendedora y que las normas subjetivas afectan directa e indirectamente a la Intención Emprendedora. Múltiples autores han utilizado el MTCP por ser buen predictor en la Intención Emprendedora, por ejemplo: Ferri et al. (2018), Tarapuez et al. (2018), Lu y Wang (2018), Johara et al. (2017), Tarapuez et al. (2015), Alonso Galicia (2012), Fayolle y Gailly (2004) y Krueger y Carsrud (1993), entre otros.

En suma, los modelos de intención son centrales para entender diferencias presentes en hombres y mujeres en cuanto a los factores que intervienen en la toma de decisiones del emprendedurista, ya que permiten predecir comportamientos sociales. Para analizar factores que se encuentran en el momento de tomar la decisión de emprender, esta investigación se consideró al igual que en el modelo de Ajzen (1991), que las intenciones para emprender dependen de las percepciones personales, las normas sociales y la viabilidad que perciben las personas.

\section{Género en el emprendimiento}

Se considera el emprendimiento desde una perspectiva de género para tomar en cuenta las diferencias en las funciones y conductas que por naturaleza pudieran crear una desigualdad al momento de emprender para los hombres y las mujeres.

Las diferencias emocionales que existen entre los hombres y las mujeres son factores que se encuentran a la hora de emprender. Por ejemplo, para las mujeres cuando la familia y la empresa se encuentran en opo- sición se manifiesta un sentimiento de culpa; en cambio, los hombres experimentan el mismo sentimiento de culpa en la idea a fracasar en el negocio. Por otra parte, la relación entre trabajo-vida familiar es un sentimiento menos intenso para los hombres (Taylor, 2017).

Su artículo explica que dentro del conjunto de normas, atributos, funciones y actividades que cada sociedad considera convenientes para las mujeres y hombres pudieran estar dejando rezagado a alguno de estos dos grupos en el tema del emprendimiento.

Muestra cómo el techo de cristal puede ser un ejemplo que enfrentan algunas mujeres en sus empleos, por lo que se vuelve un motivante para adentrarse al mundo emprendedurista (Sharma, 2020). Un ejemplo es la República Democrática del Congo, un país que cuenta con altos niveles de desempleo; esta situación lleva a las mujeres a querer crear su propia empresa; a su vez, al querer abrir un negocio, las mujeres se encuentran con barreras como los prejuicios sociales, deficiencia de servicios de apoyo, acceso limitado al financiamiento, relaciones inadecuadas, responsabilidades familiares desproporcionadas, así como tasas de educación menores (Henckel, 2017). De acuerdo con The World Bank (2019), 115 economías aún restringen legalmente a las mujeres para tener un negocio.

El mismo informe publicado en 2020 afirma que las mujeres en promedio tienen sólo las tres cuartas partes de los derechos legales otorgados a los hombres (en algunos países las mujeres no pueden firmar un contrato o abrir una cuenta bancaria) (The World Bank, 2020), por lo que el estudio muestra que las mujeres enfrentan mayores barreras al momento de querer abrir un negocio.

En general los hombres utilizan tres veces más capital que las mujeres, esto se ve reflejado en el valor de sus activos, el número de los empleados como en las ventas obtenidas (Ferri et al., 2018); por otro lado, en la región de África las empresas pertenecientes a hombres tienen seis veces más capital que las empresas pertenecientes a 
mujeres, otro factor notable en esta región son los sectores en los que la mayoría de las empresas encabezadas por mujeres se limitan a sectores tradicionalmente considerados femeninos (Gaye, 2018).

En conclusión, un factor que puede explicar las motivaciones que alientan a las mujeres a emprender desde una perspectiva de género es un sistema de seguridad social deficiente, aunque enfrenten factores desalentadores como la carga familiar desproporcionada, falta de financiamientos y prejuicios sociales.

\section{Metodología Elaboración del cuestionario}

En un estudio se encontró que de acuerdo con el colectivo, existían factores que influyen más que en otros grupos (García-Río et al., 2020), por lo que se elaboró y aplicó una encuesta a 135 alumnos de carreras afines como lo son Administración Financiera, Contabilidad, Negocios y Comercio Internacional y Actuaría de la Universidad Autónoma de Querétaro (UAQ). Se recibieron 86 encuestas completas. El cuestionario fue estructurado con preguntas de escala nominal y de escala Likert de 5 puntos desde Totalmente de acuerdo (2) a Totalmente en desacuerdo (-2).

Esta encuesta se compone de factores que inciden en la actividad emprendedora arriba analizados y está dividida en tres secciones:

1) Información general: se incluyeron preguntas sobre sus características demográficas con el objetivo de describir la muestra.

2) Factores internos que influyen en la intención de emprender: capacidad emprendedora, características del individuo (autoconfianza, innovación, autoconocimiento, etcétera), decisión de crear una empresa, predisposición a emprender, propósito de emprendimiento.

3) Factores externos que influyen en la intención de emprender: aprobación de la familia directa, amigos y compañeros; factibilidad o preparación para emprender; factores sociales y de formación; facilidad para adquirir financiamiento de la familia o del gobierno y dificultades que se pudieran presentar al momento de emprender.

\section{Construcción de variables: análisis factorial}

El análisis factorial (AF) es un método para reducir datos. El AF requiere que existan suficientes correlaciones entre las variables. Para verificarlo, se calculó un "test de esfericidad de Barlett”, el cual detecta la presencia de correlación entre variables. Si se rechaza la hipótesis nula, se asegura que el AF es adecuado para los datos. Otro indicador calculado es el índice Kaiser-Meyer-Olkin (KMO). De acuerdo con los autores, si el índice $\mathrm{KMO}$ es <0.5, no es aceptable realizar un $A F$; si $K M O$ es $\geq 0.6$ grado de correlación aceptable, y si $\mathrm{KMO}$ es $\geq 0.7$ y tiene un grado de correlación alto, es totalmente loable el AF.

El AF calcula medidas distintas a partir de las variables originales, que explican toda la varianza presente en las variables originales. El método que se utilizó para la extracción de factores es el de componentes principales. La finalidad es buscar soluciones factoriales en donde cada factor tenga altas correlaciones a un conjunto de variables pero pequeña a las demás. El método de rotación utilizado es el de Varimax, ya que disminuye el número de variables con altas cargas de un factor, haciendo mejor la interpretación de los factores.

Este cálculo permite obtener las matrices de puntuaciones factoriales (F) para cada observación. Se usó la técnica de mínimos cuadrados de acuerdo con la ecuación 1:

$$
\hat{\mathrm{F}}=\left(\mathrm{A}^{\prime} \mathrm{A}\right)^{-1} \mathrm{~A}^{\prime} \mathrm{X}
$$

\section{Especificación del modelo Logit}

A partir de los resultados del AF y la estimación de los puntos $F$, se especificó un modelo Logit para analizar los determinan- 
tes y las diferencias en la Intención Emprendedora. Esta técnica estadístico-inferencial es útil cuando la variable de respuesta $Y$ es dicotómica. En estos modelos se estima la posibilidad de que acontezca un suceso (el que suceda la Intención Emprendedora). La probabilidad se puede expresar con una función logística como se muestra en la ecuación 2 (Cameron y Trivedi, 2009):

$$
P_{i}=\frac{1}{1+e^{-\left(\alpha+\beta X_{i}\right)}}
$$

Tomando el logaritmo natural, se obtiene la razón de probabilidades (ecuación 3):

$$
\mathrm{L}_{\mathrm{i}}=\ln \left(\frac{\mathrm{P}_{\mathrm{i}}}{1-\mathrm{P}_{\mathrm{i}}}\right)=\mathrm{Z}_{\mathrm{i}}=\alpha+\beta \mathrm{X}_{\mathrm{i}}
$$

En este caso, $\beta$ mide el cambio en el logaritmo de la razón de probabilidades ocasionado por un cambio unitario en $x$. Debido a que existe una relación no lineal entre las variables explicativas y la posibilidad que acontezca un suceso, el aumento de una unidad en la variable explicativa ocasiona que los aumentos de dicha posibilidad no son siempre iguales (Cameron y Trivedi, 2009). Debido a las dificultades para interpretar los coeficientes, se calcularon los efectos marginales, como se establece en la ecuación 4:

$\frac{\delta \operatorname{Pr}\left(y_{i}=1 I x_{i}\right.}{\delta x_{i k}}=\frac{\delta F\left(x_{i}^{\prime} \beta\right)}{\delta x_{i k}}=\int\left(x_{i}^{!} \beta\right) \beta_{k}$,

En cuanto a la especificación del modelo, como se mencionó, la variable dependiente es binaria: 1 para los estudiantes que respondieron que sí tienen intención de iniciar un negocio y $\mathrm{O}$ en el caso contrario. Las variables independientes $(x)$ son los puntos $F$ calculados con el AF y las variables demográficas.

\section{Principales aspectos del cuestionario}

De los cuestionarios contestados (en adelante referidos como la muestra), del total de encuestados, $57 \%$ son mujeres y $43 \%$ son hombres. Las edades predominantes están entre 21 y 23 años y la mayor parte del alumnado se encuentra en el segundo año de su carrera (30\%) y en cuarto (37\%). Un alto porcentaje (88\%) respondió que tiene experiencia laboral previa.

En cuanto a los factores internos al momento de emprender, el alumnado contestó que quieren desarrollar su carrera profesional (50\%), crear su propia empresa (35\%) o bien trabajar en la administración pública (8\%). En relación con las características personales, el porcentaje más alto se registró para "confía en sus capacidades a pesar de que comete errores" (52\%), seguido de tener "ideas originales" (45\%) y de considerar que se tiene capacidad para "escuchar la opinión de aquellos que entienden un problema de manera diferente a la suya" (45\%). En los aspectos relativos a la determinación de iniciar una empresa, destacó la disposición a esforzarse para ser empresario (53\%) y tener como objetivo profesional ser empresario (44\%). En cuanto al propósito del emprendimiento, un alto porcentaje (78\%) lo llevaría a cabo para hacer realidad algunos sueños. Otra motivación señalada es la de tener altos ingresos económicos o ser su propio jefe (74\%).

En la tercera sección del cuestionario se preguntó acerca de los factores externos. En cuanto al apoyo financiero para emprender, destaca el de la familia (50\%), que podría facilitar locales o espacios para la empresa si los tuviera. Por último, resaltaron como dificultades para emprender la falta de capital (41\%) y la imposibilidad de obtener financiación (45\%).

En suma, el alumnado consideró que tiene capacidades para emprender, cree tener dificultades de capital y financiamiento. Dada la gran cantidad de preguntas en el cuestionario, es difícil destacar las diferencias por género, por lo que estas diferencias se analizan más adelante con la estimación del modelo Logit.

\section{Construcción de las variables}

Se procedió al análisis factorial para la construcción de las variables a partir de las respuestas de la encuesta ya reseñadas, en virtud de que el valor del índice $\mathrm{KMO}$ fue de 0.705, y el $p$ value de la prueba de es- 
fericidad de Bartlett fue menor a 0.05. ${ }^{1}$ Los factores y los autovalores se calcularon con el método de componentes principales. De acuerdo con el criterio de autovalor mayor que uno, se decidió usar los primeros 13 factores que explican $77.88 \%$ de la varianza total de las variables del cuestionario.

Del cuadro de la matriz de componentes rotados con los 13 factores, se eligieron como variables por su importancia a ocho (Características personales, Decisión de crear una empresa, Propósito del emprendimiento, Facilidad para adquirir financiamiento, Aprobación social, Miedo al fracaso, Dudas sobre si llegará a crear una empresa y Tiempo limitado para otras actividades [con la familia, la pareja, etcétera], por tener que trabajar demasiadas horas).

\section{La especificación y estimación del modelo Logit}

En estos modelos, la variable dependiente es binaria $(1,0)$; en el modelo que se propone, 1 corresponde a la probabilidad de ser emprendedurista.

Como variables independientes se tomaron, además de los ocho factores ya calculados, cuatro variables más asociadas con factores demográficos que también inciden en el emprendimiento.

\section{Resultados}

En la tabla 1 se exhiben los resultados de la estimación del modelo Logit para el alumnado. Para evitar los posibles problemas de heterocedasticidad, la estimación se realizó con el método de errores robustos. Los resultados fueron satisfactorios, ya que en conjunto, los coeficientes estimados fueron estadísticamente significativos con base en la prueba de Wald ( $p$ value menor 0.05) y la prueba de Pseudo R-cuadrada con un valor de 0.29. Por último, el porcentaje de los valores correctamente clasificados es de $74.42 \%$.

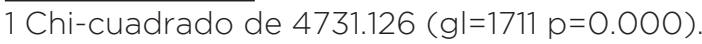

Tabla 1

\section{Modelo Logit: Global}

\begin{tabular}{|l|c|c|}
\hline \multicolumn{1}{|c|}{ Intención Emprendedora } & Coeficiente & P>IzI \\
\hline Género & -1.24 & $0.07^{*}$ \\
\hline Año & & \\
\hline 3 & 0.59 & 0.49 \\
\hline 4 & 0.97 & 0.17 \\
\hline Experiencia laboral previa & 1.62 & $0.06^{*}$ \\
\hline Empresarios en la familia & -1.93 & $0.04^{* *}$ \\
\hline Características personales & -0.65 & $0.01^{* * *}$ \\
\hline Decisión de crear una empresa & 1.64 & $0.00^{* * *}$ \\
\hline Propósito del emprendimiento & -0.63 & $0.03^{* *}$ \\
\hline $\begin{array}{l}\text { Facilidad para adquirir } \\
\text { financiamiento }\end{array}$ & 0.61 & $0.05^{* *}$ \\
\hline Aprobación social & -0.32 & 0.31 \\
\hline $\begin{array}{l}\text { Tiempo limitado para otras } \\
\text { actividades }\end{array}$ & 0.28 & 0.53 \\
\hline $\begin{array}{l}\text { Miedo al fracaso } \\
\text { Dudas sobre si llegará a crear }\end{array}$ & -0.92 & $0.01^{* * *}$ \\
\hline una empresa & -0.38 & 0.67 \\
\hline cons & & 0.38 \\
\hline p<0.1; **p<0.05 ***<0.01 & & \\
\hline
\end{tabular}

Wald chi2(13) $=24.07$ Prob $>$ chi2 $=0.03$.

Pseudo R2=0.29

Fuente: elaboración propia a partir de los reportes del análisis STATA.

Se observó que los coeficientes que resultaron estadísticamente significativos con signo positivo son: Experiencia laboral previa, Decisión de crear una empresa, Características personales de un emprendedurista y Facilidad para adquirir financiamiento; lo que significa que estas variables aumentan la probabilidad de emprender.

Los coeficientes con signo negativo fueron: Género, Empresarios en la familia, Propósito del emprendimiento y Dudas sobre si llegará a crear una empresa; al contrario de las anteriores, estas variables disminuyen la probabilidad de emprender.

En virtud de que los coeficientes no permiten analizar la magnitud en la cual las variables inciden en la probabilidad de emprender, se estimaron los efectos marginales que se reportan en la tabla 2 . Se observó que ser mujer disminuye la probabilidad de emprender en 20 puntos porcentuales. 
Concordó con la afirmación de varios autores (Sánchez-Torné et al., 2021; Molino et al., 2018; Amorós y Abarca, 2014; Fuentes García y Sánchez Cañizares, 2010; García Ramos et al., 2010), en cuanto a que existe una superioridad en la intención de iniciar un negocio en los hombres comparada con la de las mujeres, ya que ellas muestran una menor predisposición a emprender.

Contar con experiencia laboral previa aumentó la probabilidad en la intención de emprender en 26 puntos porcentuales. Ferri et al. (2018) y Espíritu Olmos (2011) explicaron que la experiencia laboral da al emprendedurista información y herramientas para poder evaluar riesgos y oportunidades.

Cuando hay empresarios en la familia del alumno disminuye la probabilidad de emprender en 31 puntos porcentuales. Este resultado difirió del de Belas et al. (2017), Amorós y Abarca (2014), Arenius y Minniti (2005), Fuentes García y Sánchez Cañizares (2010) y García Ramos et al. (2010). Estos autores explican que tanto mujeres como hombres que conocen a empresarios sienten que tienen la capacidad de emprender, perciben que existen buenas oportunidades, estiman que cuentan con habilidades y conocimiento, por lo que sus posibilidades de emprender son mayores en comparación con los que no tienen empresarios en su familia. Por otro lado, esta discrepancia puede ser explicada con la "teoría del aprendizaje social", ya que el individuo toma las experiencias y opiniones positivas o negativas de seres cercanos que lo incentiven o desincentiven para emprender (Echeverri-Sánchez et al., 2018).

Contrariamente a lo esperado, las características emprendedoras personales del individuo disminuyen la probabilidad de emprender en 10 puntos porcentuales. García Ramos et al. (2010) señalan que las características personales aumentan la probabilidad de emprender. También afirman que las atribuciones particulares del individuo que crea una empresa son distintas a aquellas de las demás personas. Dicho antagonismo en el resultado posiblemente se deba a que el alumnado se sienta inexperto e inseguro, y por ello las características emprendedoras personales registraron un efecto negativo en la probabilidad de emprender.

La decisión de iniciar un negocio aumenta la probabilidad de la Intención Emprendedora en 26 puntos porcentuales; lo cual parece comprobar la noción de Liñán (2007) de que el desarrollo para crear una empresa empieza en el momento donde toma la decisión de crearla.

El propósito del emprendimiento disminuyó la probabilidad de emprender en 10 puntos porcentuales; esto es contrario a lo señalado por Osorio y Londoño (2016), quienes señalan que la Intención Emprendedora se puede medir en términos de que el individuo tenga claro el propósito del emprendimiento y que resulta insuficiente el deseo de ser emprendedurista, más bien se requiere una visión que permita lograr el objetivo. El resultado negativo no esperado pudo deberse a la falta de claridad en el propósito de emprender por parte del alumnado. La facilidad para adquirir financiamiento aumenta la probabilidad de la Intención Emprendedora en 10 puntos porcentuales; lo cual concuerda con varios autores: Hernández-López et al. (2018), Belas et al. (2017), Alonso Galicia (2012) y Liñán (2004) en cuanto a señalar la importancia de los apoyos financieros o de recursos en la creación de una empresa. Por último, las dudas que tuvo el individuo sobre si alguna vez llegará a crear una empresa disminuyeron la probabilidad de emprender en 15 puntos porcentuales; lo que coincidió con lo encontrado con Belas et al. (2017), McMullen y Shepherd (2006) y Ajzen (1991). Los autores sostienen que las dudas de los individuos sobre la creación de un negocio influyen negativamente en la Intención Emprendedora. Si se encontraran dos individuos con la misma fuerza de Intención Emprendedora, pero uno de ellos tuviera la duda de si alguna vez llegará a lograrlo, este último tendrá menos probabilidades de emprender. A su vez, el hecho de tener las oportunidades emprendedoras a la mano no propiciará necesariamente el emprendimiento, ya que las dudas podrían llegar a presentarse. 
Tabla 2 Efectos marginales

\begin{tabular}{|l|c|c|}
\hline \multicolumn{1}{c|}{ Intención Emprendedora } & $\boldsymbol{d y} / \boldsymbol{d x}$ & $\boldsymbol{P > z}$ \\
\hline Género & $-0.20^{* *}$ & 0.04 \\
\hline Año & & \\
\hline 3 & 0.09 & 0.48 \\
\hline 4 & 0.15 & 0.17 \\
\hline Experiencia laboral previa & $0.26^{* *}$ & 0.04 \\
\hline Empresarios en la familia & $-0.31^{* *}$ & 0.02 \\
\hline Características personales & $-0.10^{* * *}$ & 0.01 \\
\hline Decisión de crear una empresa & $0.26^{* * *}$ & 0.00 \\
\hline Propósito del emprendimiento & $-0.10^{* *}$ & 0.02 \\
\hline Facilidad para adquirir financiamiento & $0.10^{* *}$ & 0.03 \\
\hline Aprobación social & -0.05 & 0.29 \\
\hline Tiempo limitado para otras actividades & -0.03 & 0.52 \\
\hline Miedo al fracaso & 0.04 & 0.36 \\
\hline Dudas sobre si llegará a crear una & $-0.15^{* * *}$ & 0.00 \\
\hline empresa & & \\
\hline
\end{tabular}

Fuente: elaboración propia a partir de los reportes del análisis STATA.

Con la intención de analizar algunos escenarios y observar diferencias en la intención de desarrollar una empresa en las mujeres y los hombres, se calcularon los valores marginales para algunos valores representativos. El primer escenario se calculó con las variables que resultaron ser estadísticamente significativas: Experiencia laboral previa, Empresarios en la familia, Características personales del individuo, Decisión de crear una empresa, Propósito del emprendimiento, Facilidad para adquirir financiamiento y Dudas sobre si llegará a crear una empresa (dándoles valores por arriba del promedio). La probabilidad de Intención Emprendedora obtenida con este escenario fue de $46.60 \%$ para los hombres y de $21.64 \%$ para las mujeres (ver Anexo A). En un segundo escenario se tomaron las variables con influencia positiva, como Experiencia laboral previa, Decisión de crear una empresa y Facilidad para adquirir financiamiento (dándoles valores por arriba del promedio). Se obtuvo una alta probabilidad en los hombres (84.93\%), mientras que para las mujeres la probabilidad fue de $67.60 \%$ (ver Anexo B).
En todos los escenarios presentados, las mujeres mostraron tener una menor probabilidad de Intención Emprendedora que los hombres.

\section{Modelo para mujeres y para hombres}

Para seguir analizando las diferencias en la Intención Emprendedora por género, se estimó un modelo Logit para los hombres y otro para las mujeres. El porcentaje de los valores correctamente clasificados en el modelo de los hombres es de $83.78 \%$ y en el de las mujeres es de $73.47 \%$. En la tabla 3 se presenta el producto de la estimación del modelo Logit de ambos modelos. Se puede ver que en el de las mujeres, las variables significativas y con una influencia positiva en la Intención Emprendedora fueron: Encontrarse en el cuarto año de la carrera y Decisión de crear una empresa. Las variables significativas, pero con una influencia negativa en la Intención Emprendedora, fueron: Características personales, Propósito del emprendimiento y Dudas sobre si llegará a crear una empresa. Para los hombres, resultaron significativas y con una influencia negativa en la Intención Emprendedora las variables: Empresarios en la familia y Dudas sobre si llegará a emprender. Las variables con influencia positiva en la Intención Emprendedora fueron: Decisión de crear una empresa y Facilidad para adquirir financiamiento.

\section{Tabla 3}

\section{Regresión Logit para mujeres y hombres}

\begin{tabular}{|c|c|c|c|c|}
\hline \multirow[b]{2}{*}{ Variable } & \multicolumn{2}{|c|}{ Hombres } & \multicolumn{2}{|c|}{ Mujeres } \\
\hline & $\begin{array}{l}\text { Coefi- } \\
\text { ciente }\end{array}$ & $P>Z$ & $\begin{array}{l}\text { Coefi- } \\
\text { ciente }\end{array}$ & $P>Z$ \\
\hline \multicolumn{5}{|l|}{ Año } \\
\hline 3 & -3.18 & 0.34 & 1.07 & 0.30 \\
\hline 4 & 0.63 & 0.78 & $2.15^{* *}$ & 0.02 \\
\hline Empresarios en la familia & $\left|-7.10^{* * *}\right|$ & 0.00 & 1.41 & 0.18 \\
\hline Características personales & $1.03^{* *}$ & 0.53 & -0.98 & 0.02 \\
\hline $\begin{array}{l}\text { Decisión de crear una } \\
\text { empresa }\end{array}$ & $6.43^{*}$ & 0.06 & $1.35^{*}$ & 0.07 \\
\hline $\begin{array}{l}\text { Propósito del } \\
\text { emprendimiento }\end{array}$ & -0.48 & 0.80 & $-0.75^{* *}$ & 0.05 \\
\hline
\end{tabular}


Tabla 3 (continuación)

\begin{tabular}{l|c|c|c|c}
\hline $\begin{array}{l}\text { Facilidad para adquirir } \\
\text { financiamiento }\end{array}$ & $2.10^{* *}$ & 0.05 & 0.26 & 0.61 \\
\hline $\begin{array}{l}\text { Aprobación social } \\
\begin{array}{l}\text { Tiempo limitado para otras } \\
\text { actividades }\end{array}\end{array}$ & -0.01 & 0.99 & -0.60 & 0.17 \\
\hline $\begin{array}{l}\text { Miedo al fracaso } \\
\begin{array}{l}\text { Dudas sobre si llegará a } \\
\text { crear una empresa }\end{array}\end{array}$ & -1.34 & 0.32 & 0.19 & 0.62 \\
\hline cons & 4.10 & 0.10 & -0.16 & 0.71 \\
\hline
\end{tabular}

Fuente: elaboración propia a partir de los reportes del análisis STATA.

A partir de los modelos anteriores, se calcularon los efectos marginales (tabla 4). En el modelo de los hombres se encontró que la Decisión de crear una empresa y la Facilidad para adquirir financiamiento aumentan la Intención Emprendedora en 55 y 18 puntos porcentuales, respectivamente. Por el contrario, la Duda sobre si llegarán a crear una empresa y la existencia de Empresarios en su familia disminuyeron la Intención Emprendedora en 16 y 61 puntos porcentuales, respectivamente.

El modelo de las mujeres arrojó que Cursar el cuarto año de la carrera y la Decisión de crear una empresa aumentan la Intención Emprendedora en 32 y 21 puntos porcentuales, respectivamente. Por otro lado, las Características personales, Dudas sobre si llegará a crear una empresa y el Propósito del emprendimiento disminuyeron la probabilidad de emprender en 15, 14 y 11 puntos porcentuales, respectivamente.

En suma, sólo hubo coincidencia en las variables Decisión de crear una empresa y Dudas sobre si llegará a crear una empresa. La variable Decisión de crear una empresa aumenta la probabilidad de emprender de los hombres. La Facilidad para adquirir financiamiento no fue significativa para las mujeres, pero sí lo fue Cursar el cuarto año de la carrera, esto pudo deberse que a medida que avanzan en el año escolar incrementa su Intención Emprendedora (Arteaga Cervantes et al., 2016). Por otro lado, las Características personales afectaron de manera negativa la intención de desarrollar una empresa en las mujeres; esto pudo deberse a que su percepción de estas características fue ligeramente más baja en comparación con la autopercepción de los hombres. Así, mostró que, en efecto, existen diferencias en los factores que inciden en la Intención Emprendedora por género, y que las mujeres enfrentan mayores dificultades que los hombres.

Dentro de los resultados no esperados, estaba el signo negativo en Empresarios en la familia dentro del modelo de los hombres. Recordando a Echeverri-Sánchez et al. (2018), esto se pudo explicar con la teoría del aprendizaje social, que señala que el alumnado puede estar tomando de su familia experiencias/opiniones (probablemente negativas) que lo desmotivan para emprender.

En cuanto a las mujeres existió un signo negativo no esperado en las Características emprendedoras personales del individuo, ya que disminuyeron la probabilidad de emprender. Esto pudo deberse a que las alumnas reflejaron en el reconocimiento de sus características emprendedoras (confianza en sí mismas a pesar de cometer errores, creer firmemente en tener éxito, adaptación fácil a los cambios, etcétera) una menor autoestima, la cual disminuyó las intenciones emprendedoras. Un estudio explicó que existe una relación significativa y positiva entre el alumnado que manifiesta una superior percepción de sí mismo con la Intención Emprendedora (Espíritu Olmos et al. 2012). Este hecho también lo confirmó Taylor (2017), quien explicó que el espíritu empresarial masculino se relaciona con atributos como "la autoestima, la asunción de riesgos, la toma de decisiones autónoma, el exceso de confianza, la necesidad de control, la resiliencia y el ego", lo que no sucede con el espíritu empresarial femenino.

El propósito del emprendimiento disminuyó la probabilidad de emprender en las mujeres, contrario a lo que explica la literatura (Osorio y Londoño, 2016). Este resultado no esperado se podría explicar con el hecho de que las mujeres no se encuentran identificadas con un propósito emprendedurista o no tienen claridad en el propósito de emprender en la etapa universitaria en la que están. 
En este estudio no resultó significativo como un factor que incentive la Intención Emprendedora la Aprobación social. Algunos autores afirman que los individuos son más proclives a emprender si perciben que existe una aprobación social adecuada (Alonso Galicia et al., 2019; Ferri et al., 2018; Hernández-López et al., 2018; Jiménez-Cercado et al., 2017; Alonso Galicia, 2012; Liñán, 2004; Ajzen, 1991). A su vez, tampoco resultaron significativas las variables Miedo al fracaso ni el Tiempo limitado para otras actividades. Resultados similares en cuanto a la relación de estas variables y la Intención Emprendedora se encuentran en varios estudios (Alonso Galicia et al., 2019; García Ramos et al., 2010, Belas et al., 2017; The World Bank, s.f.).

\section{Tabla 4}

\section{Efectos marginales para el análisis de las mujeres y los hombres}

\begin{tabular}{|c|c|c|c|c|}
\hline \multirow{2}{*}{ Variable } & \multicolumn{2}{|c|}{ Hombres } & \multicolumn{2}{|c|}{ Mujeres } \\
\hline & $d y / d x$ & $P>Z$ & $d y / d x$ & $P>Z$ \\
\hline \multicolumn{5}{|l|}{ Año } \\
\hline 3 & -0.24 & 0.24 & 0.15 & 0.29 \\
\hline 4 & 0.05 & 0.80 & $0.32^{* * *}$ & 0.01 \\
\hline $\begin{array}{l}\text { Empresarios en la fa- } \\
\text { milia }\end{array}$ & $-0.61^{* * *}$ & 0.02 & 0.22 & 0.18 \\
\hline $\begin{array}{l}\text { Características perso- } \\
\text { nales }\end{array}$ & 0.09 & 0.50 & $-0.15^{* * *}$ & 0.01 \\
\hline $\begin{array}{l}\text { Decisión de crear una } \\
\text { empresa }\end{array}$ & $0.55^{* * *}$ & 0.00 & $0.21^{* *}$ & 0.06 \\
\hline $\begin{array}{l}\text { Propósito del empren- } \\
\text { dimiento }\end{array}$ & -0.04 & 0.81 & $-0.11^{* * *}$ & 0.04 \\
\hline $\begin{array}{l}\text { Facilidad para adquirir } \\
\text { financiamiento }\end{array}$ & $0.18^{* * *}$ & 0.00 & 0.04 & 0.61 \\
\hline Aprobación social & 0.00 & 0.99 & -0.09 & 0.15 \\
\hline $\begin{array}{l}\text { Tiempo limitado para } \\
\text { otras actividades }\end{array}$ & -0.07 & 0.29 & 0.03 & 0.62 \\
\hline Miedo al fracaso & 0.12 & 0.28 & -0.02 & 0.71 \\
\hline $\begin{array}{l}\text { Dudas sobre si llegará } \\
\text { a crear una empresa }\end{array}$ & $-0.16^{* * *}$ & 0.04 & $-0.14^{* * *}$ & 0.03 \\
\hline
\end{tabular}

Fuente: elaboración propia a partir de los reportes del análisis STATA.

En un primer escenario para los hombres con todas las variables que fueron significativas (Empresarios en la familia, Decisión de crear una empresa, Facilidad para adquirir financiamiento y Duda sobre si llegará a crear una empresa), la probabilidad de Intención Emprendedora fue de 88.67\% (ver Anexo C). En un segundo escenario, se estimaron en el modelo las variables que resultaron significativas para las mujeres (Cuarto año de la carrera, Características personales, Decisión de crear una empresa, Propósito del emprendimiento y Duda sobre si llegará a crear una empresa); en él, la probabilidad de Intención Emprendedora fue de $21.20 \%$ (ver Anexo D).

Por último, se compararon entre hombres y mujeres sólo las variables que influyeron de forma positiva (mujeres: Cuarto año de la carrera y Decisión de crear una empresa, Hombres: Decisión de crear una empresa y Facilidad para adquirir financiamiento). La Intención Emprendedora para ellos fue de $94.19 \%$, mientras que para las mujeres fue de $69.22 \%$. En suma, se observó que la probabilidad de emprender es menor para las mujeres bajo todos los escenarios considerados (ver Anexo E).

\section{Conclusiones}

La actividad de las mujeres en el área emprendedora ha ido en aumento, pero la brecha entre mujeres y hombres aún es amplia. La revisión de la literatura encontrada y el modelo realizado ponen en evidencia que los factores internos, externos y algunas variables demográficas son determinantes en la Intención Emprendedora. Se piensa que los factores para emprender un negocio afectan de igual manera a mujeres y hombres; sin embargo, no siempre es así. Desconocerlo implica un sesgo en las políticas públicas y programas de emprendimiento.

Estos resultados muestran las diferencias en los factores que se encuentran en la probabilidad de iniciar un negocio en hombres y mujeres; entre ellas se encuentran las siguientes: a medida que las mujeres avanzan en el estudio de su carrera, su Intención Emprendedora aumenta. En el caso de los hombres, esta variable resultó no ser relevante. Las características personales inciden nega- 
tivamente en las mujeres. Una posible explicación es que las alumnas tengan una menor autoestima y por ello se perciban como menos capaces de iniciar un negocio.

De manera similar al caso anterior, el propósito del emprendimiento también influye negativamente en la Intención Emprendedora femenina debido, en parte, a que las alumnas aún no tienen clara la razón para emprender.

Para los hombres, el hecho de que haya empresarios en su familia disminuye su Intención Emprendedora. Quizás esto se deba a que los alumnos están influidos por experiencias/opiniones negativas de personas cercanas que los desincentivan para iniciar un negocio.

La variable Facilidad para adquirir financiamiento es significativa y positiva en los hombres, lo cual sostiene lo expuesto en la literatura, pues existe una percepción general de que los hombres acceden a más fuentes de financiamiento y capital que las mujeres.

Dentro de las similitudes encontradas están la influencia positiva que tiene en la Intención Emprendedora la decisión de iniciar una empresa y la influencia negativa que produce la duda sobre si llegará a crear una empresa.

Como era esperado en todos los escenarios calculados, las mujeres cuentan con menores probabilidades que los hombres de emprender un negocio. Este resultado alude a que ellas perciben mayores dificultades para emprender debido a sus propias percepciones sobre el entorno y posibles malas experiencias. De aquí la necesidad de políticas públicas, programas y currículos de fomento al emprendimiento con un enfoque de género que tomen en consideración estas diferencias que existen en la intención de iniciar un negocio en hombres y mujeres.

Una limitante en esta investigación es que la población de estudio fue de una sola universidad y de carreras afines a la Administración y Contaduría, por lo que se debe tener precaución al extrapolar dichas conclusiones a otros contextos. Dejamos para futuras investigaciones realizar este ejercicio con una muestra más grande y heterógena con alumnos de diferentes carreras y universidades. No obstante, a pesar de esta limitación consideramos que los resultados son congreuntes con estudios previos sobre el tema.

\section{Anexos}

\section{Anexo A}

\section{Primer escenario del modelo global}

\section{Escenario en hombres}

Experiencia laboral previa, Empresarios en la familia, Características personales, Decisión de crear una empresa, Propósito del emprendimiento, Facilidad para adquirir financiamiento y Dudas sobre si llegará a crear una empresa

\begin{tabular}{c|c|c|c|c|c|c} 
& Margen & Método delta Std. Err & $z$ & $P>z$ & {$[95 \%$ Conf. } & Intervalo] \\
\multirow{2}{*}{ _cons } & 0.465999 & 0.1590338 & 2.93 & 0.003 & 0.1542985 & 0.7776995 \\
\multicolumn{4}{|c|}{ Escenario en mujeres }
\end{tabular}

Experiencia laboral previa, Empresarios en la familia, Características personales, Decisión de crear una empresa, Propósito del emprendimiento, Facilidad para adquirir financiamiento y Dudas sobre si llegará a crear una empresa

\begin{tabular}{c|c|c|c|c|c|c} 
& Margen & Método delta Std. Err & $z$ & P> z & [95\% Conf. & Intervalo] \\
\hline \multirow{2}{*}{ _cons } & 0.2163856 & 0.1278327 & 1.69 & 0.091 & -0.0341618 & 0.466933 \\
\hline
\end{tabular}

Fuente: elaboración propia a partir de los reportes del análisis STATA. 


\section{Anexo B Segundo escenario del modelo global}

\section{Escenario en hombres}

Experiencia laboral previa, Decisión de crear una empresa y Facilidad para adquirir financiamiento

\begin{tabular}{c|c|c|c|c|c|c} 
& Margen & Método delta Std. Err & $z$ & $P>z$ & {$[95 \%$ Conf. } & Intervalo] \\
_cons & 0.8493042 & 0.0672752 & 12.62 & 0 & 0.7174473 & 0.9811612
\end{tabular}

Experiencia laboral previa, Decisión de crear una empresa y Facilidad para adquirir financiamiento

\begin{tabular}{c|c|c|c|c|c|c} 
& Margen & Método delta Std. Err & $z$ & $P>z$ & {$[95 \%$ Conf. } & Intervalo] \\
\hline \multirow{2}{*}{ cons } & 0.6759706 & 0.09397 & 7.19 & 0 & 0.4917928 & 0.8601485 \\
\hline
\end{tabular}

Fuente: elaboración propia a partir de los reportes del análisis STATA.

\section{Anexo C Escenario hombres}

\section{Escenario en hombres}

Empresarios en la familia, Decisión de crear una empresa, Facilidad para adquirir financiamiento y Duda sobre si llegará a crear una empresa

\begin{tabular}{c|c|c|c|c|c|c} 
& Margen & Método delta Std. Err & $z$ & $P>z$ & {$[95 \%$ Conf. } & Intervalo] \\
\hline _cons & .8867403 & .0963924 & 9.20 & 0.000 & .6978147 & 1.075666 \\
\hline
\end{tabular}

Fuente: elaboración propia a partir de los reportes del análisis STATA.

\section{Anexo D Escenario mujeres}

\section{Escenario en mujeres}

Cuarto año de la carrera, Características personales, Decisión de crear una empresa, Propósito del emprendimiento y Duda sobre si llegará a crear una empresa

\begin{tabular}{c|c|c|c|c|c|c} 
& Margen & Método delta Std. Err & $z$ & $\mathrm{P}>\mathrm{z}$ & [95\% Conf. & Intervalo] \\
\hline _cons & .2120313 & .1538164 & 1.38 & 0.168 & -.0894433 & .513506 \\
\hline
\end{tabular}

Fuente: elaboración propia a partir de los reportes del análisis STATA.

\section{Anexo E Escenario: factores positivos para ellos y para ellas}

\section{Escenario en hombres}

Decisión de crear una empresa y Facilidad para adquirir financiamiento

\begin{tabular}{c|c|c|c|c|c|c} 
& Margen & Método delta Std. Err & z & P> z & {$[95 \%$ Conf. } & Intervalo] \\
_cons & .9418633 & .0305743 & 30.81 & 0.000 & .8819387 & 1.001788
\end{tabular}

Cuarto año de la carrera y Decisión de crear una empresa

\begin{tabular}{c|c|c|c|c|c|c} 
& Margen & Método delta Std. Err & $z$ & $P>z$ & {$[95 \%$ Conf. } & Intervalo] \\
\hline _cons & .6921918 & .1279024 & 5.41 & 0.000 & .4415077 & .942876 \\
\hline
\end{tabular}

Fuente: elaboración propia a partir de los reportes del análisis STATA. 


\section{Fuentes consultadas}

Ajzen, Icek (1991), "The theory of planned behavior", Organizational Behavior and Human Decision Processes, 50 (2), Ámsterdam, Elsevier, pp. 179-211, doi: https://doi.org/10.1016/0749-5978(91)90020-T

Alonso Galicia, Patricia Esther; Barrera Badillo, Patricia Angélica; Barrón Jiménez, Samantha; Berrelleza Rendón, Mildred Daniela; Carbajal Marrón, Moisés; Cortés Vázquez, Judith; Jiménez Rodríguez, Víctor Manuel; Pouzou, Romain y Rehák, Ján (2019), El emprendimiento en el Estado de Querétaro. Análisis de los datos del Global Entrepreneurship Monitor 2019/2020, Querétaro, Global Entrepreneurship Research Association/Tecnológico de Monterrey, Campus Querétaro, <https://bit.ly/3B5X$\mathrm{HRr}>, 2$ de marzo de 2020.

Alonso Galicia, Patricia Esther (2012), "La configuración de la Intención Emprendedora entre académicos responsables de proyectos de investigación en España. Un enfoque de género", tesis de doctorado, Universidad de Cantabria, Santander.

Amorós, José Ernesto y Abarca, Adriana (2014), "Global Entrepreneurship Monitor: Reporte Nacional de Chile 2014", Santiago de Chile, Universidad del Desarrollo, <https://bit.ly/3vJcbpA>, 8 de noviembre de 2019.

Arboleda, María Elvira (2014), "Emprendimiento y tecnología, una oportunidad de vida para la mujer colombiana", Revista de Economía \& Administración, 11 (2), Cali, Universidad Autónoma de Occidente, pp. 19-29, <https://bit.ly/3Efs22e>, 16 de diciembre de 2019.

Arenius, Pia y Minniti, Maria (2005), "Perceptual Variables and Nascent Entrepreneurship", Small Business Economics, 24 (3), Luxemburgo, Springer Science/ Business Media pp. 233-247, < https:// bit.ly/3Cilmiis, 16 de diciembre de 2019.
Arteaga Cervantes, Ana Laura; Mexicano Ojeda, Mario Alberto y González Martínez, Rocío (2016), "Intención emprendedora en estudiantes de la licenciatura de administración en una institución de educación superior", ponencia presentada en el XXI Congreso Internacional de Contaduría, Administración e Informática, 5-7 de octubre, Ciudad de México.

Belas, Jaroslav; Gavurová, Beáta; Schonfeld, Jaroslav; Zvarikova, Katarina y Kacerauskas, Tomas (2017), "Social and economic factors affecting the entrepreneurial intention of university students", Transformations in Business and Economics, 16 (3), Kaunas, Vilnius University, pp. 220-239, <https://bit.ly/ 31j6bZK>, 1 de abril de 2019.

Bolio, Eduardo; Garza, Gabriela; Ibarra, Valentina y Rentería, Melissa (2018), One aspiration, two realities. Promoting gender equality in Mexico, Ciudad de México, Mckinsey \& Company, <https://mck.co/3OPZbmt>, 1 de noviembre de 2019 .

Cameron, Colin A., y Trivedi, Pravin K. (2009), Microeconometrics Using Stata, College Station, Stata Press.

Echeverri-Sánchez, Laura; Valencia-Arias, Alejandro; Benjumea-Arias, Martha y Barrera-Del Toro, Andri (2018), "Factores que inciden en la intención emprendedora del estudiantado universitario: Un análisis cualitativo", Revista Electrónica Educare (Educare Electronic Journal), 22 (2), Heredia, Universidad Nacional, pp. 1-19, doi: https://doi. org/10.15359/ree.22-2.10, 8 de noviembre de 2019 .

Espíritu Olmos, Roberto (2011), "Análisis de la intención emprendedora en estudiantes universitarios a través de los rasgos de personalidad", Multiciencias, 11 (1), Punto Fijo, Universidad de Zulia, pp. 65-75, <https://bit.ly/3pzbfTl>, 18 de octubre de 2019.

Espíritu Olmos, Roberto; Moreno Zacarías, Hugo Martín y Priego Huertas, Héctor 
(2012), "Rasgos de personalidad innovación y autoestima en la intención emprendedora de estudiantes universitario", Multiciencias, 12, Punto Fijo, Universidad de Zulia, pp. 52-58, <https:// bit.ly/3B50RVK>, 3 de diciembre de 2019.

Fayolle, Alain y Gailly, Benoît (2004), "Using the Theory of Planned Behaviour to Assess Entrepreneurship Teaching Programs: A First Experimentation", trabajo presentado en la 14th Annual IntEnt, 4-7 de julio, University of Napoli Federico II, Italia, <https://bit.ly/3ISYOKN>, 10 de diciembre de 2021.

Ferri, Luca; Ginesti, Gianluca; Spanò, Rosanna y Zampella, Annamaria (2018), "Exploring the Entrepreneurial Intention of Female Students in Italy", Journal of Open Innovation: Technology, Market, and Complexity, 4 (3), Basilea, MDPI Multidisciplinary Digital Publishing Institute, pp.1-10, doi: https://doi. org/10.3390/joitmc4030027

Fuentes García, Fernando J. y Sánchez Cañizares, Sandra Ma. (2010), "Análisis del perfil emprendedor una perspectiva de género", Estudios de economía aplicada, 28 (3), Almería, Universidad de Almería, pp. 1-28, <https://bit.ly/3CnBkby>, 7 de octubre de 2019.

García Ramos, Constantino; Martínez Campillo, Almudena y Fernández Gago, Roberto (2010), "Características del emprendedor influyentes en el proceso de creación empresarial y en el éxito esperado", Revista Europea de Dirección y Economía de la Empresa, 19 (2), Vigo, Academia Europea de Dirección y Economía de la Empresa, pp. 31-48, <https://bit.ly/3nrHIZc>, 10 de noviembre de 2019.

García-Río, Esther; Baena-Luna, Pedro; Sánchez-Torné, Isadora y Pérez-Suárez, Macarena (2020), "Entrepreneurial Intentions determinants elements in university students. A study from a Gender Perspective", 3C Empresa. Investigación y Pensamiento Crítico, 9 (1), Alcoy, Área de Innovación y Desarro-
Ilo, S.L. pp. 89-107, doi: https://doi.org/10.17993/3cemp.2020.090141.89-107

Gaye, Diariétou (2018), "Female Entrepreneurs: the Future of the African Continent", Washington, D.C., World Bank, <https://bit.ly/3Egn2LO>, 17 de febrero de 2019.

Hernández López, Diana Milena; Moncada-Toro, John Fredy y Henao-Colorado, Laura Cristina (2018), "Intención emprendedora de los empleados del sector privado de la ciudad de Medellín y su área metropolitana", Revista CEA, 4 (8), Medellín, Institución Universitaria ITM, pp. 13-33, doi: https://doi. org/10.22430/24223182.1045

Jiménez-Cercado, María E.; Villacís-Aveiga, Washington $\mathrm{H}$. y Acosta-Veliz, Marjorie M. (2017), "Factores que conforman la intención emprendedora de estudiantes de la Universidad de Guayaquil", Revista Científica Dominio de las Ciencias, 3 (3), Guayaquil, Polo de Capacitación, Investigación y Publicación, pp. 1116-1146, <https://bit.ly/3dD4A3s>, 10 de diciembre de 2021.

Johara, Fatema; Yahya, Sofri Bin y Tehseen, Shehnaz (2017), "Determinants of Future Entrepreneurship and Entrepreneurial Intention", Global Business and Management Research: An International Journal, 9 (4), Boca Ratón, Universal Publishers, pp. 80-95, <https://bit. |y/3|9|r|5>, 10 de diciembre de 2021.

Kets de Vries, Manfred F. R. (1977), "The entrepreneurial personality: a person at the crossroads", Journal of Management Studies, 14 (1), Durham City, Durham Business School Mill Hill Lane, pp. 34-57, doi: https://doi.org/10.1111/j.1467-6486.1977.tb00616.x

Krueger, Norris F. y Carsrud, Alan L. (1993), "Entrepreneurial intentions: Applying the theory of planned behaviour", Entrepreneurship \& Regional Development, 5 (4), Londres, Taylor \& Francis, pp. 315-330, doi: https://doi. org/10.1080/08985629300000020 
Liñán, Francisco (2007), "The role of entrepreneurship education in the entrepreneurial process", en Alain Fayolle (ed.), Handbook of Research in Entrepreneurship Education, vol. 1, Cheltenham, Center for Innovation and Entrepreneurship Activities/University of Cagliari, pp. 230-247, <https://bit. ly/3puFwmx>, 7 de diciembre 2019.

Liñán, Francisco (2004), "Intention-based models of entrepreneurship education", Boston, Research Gate, pp. 1-30, <https://bit.ly/3Cq1bA1>, 29 de noviembre 2019.

Lu, Hong y Wang, Jiafure (2018), "Entrepreneurial Intention of Two Patterns of Planned Behaviour and Alertness: Empirical Evidence in China", The Journal of Asian Finance, Economics and Business, 5 (2), Daejeon, Korea Institute of Science and Technology Information, pp. 63-72, doi: https://doi.org/10.13106/ jafeb.2018.vol5.no2.63

McMullen, Jeffery S., y Shepherd, Dean A. (2006), "Entrepreneurial action and the role of uncertainty in the theory of the entrepreneur", Academy of Management Review, 31 (1), Nueva York, AOM, pp. 132-152, doi: https://doi. org/10.5465/amr.2006.19379628

Molino, Monica; Dolce, Valentina; Cortese, Claudio Giovanni y Ghislieri, Chiara (2018), "Personality and social support as determinants of entrepreneurial intention. Gender differences in Italy", PLOS ONE, 13 (6), San Francisco, PLOS, e0199924, doi: https://doi.org/10.1371/ journal.pone.0199924

Osorio, Fernando Fabián y Londoño Roldán, Juan Carlos (2016), "Intención emprendedora de estudiantes de educación media: extendiendo la teoría de comportamiento planificado mediante el efecto exposición", Cuadernos de Administración, 28 (51), Bogotá, Pontificia Universidad Javeriana, pp. 103-131, doi: https://doi.org/10.11144/Javeriana. cao28-51.ieee
Pereira Laverde, Fernando (2003), "Reflexión sobre algunas características del espíritu emprendedor colombiano", Econ. Gest. Desarro., 1, Cali, pp. 9-26, <https://bit.ly/31cXT5O>, 19 de abril de 2019.

Pfeilstetter, Richard (2011), "El emprendedor. Una reflexión crítica sobre usos y significados actuales de un concepto", Gazeta de Antropología, 27 (1), Jaén, Universidad de Jaén, pp. 1-11, <https://bit.ly/3m7VvEO>, 19 de diciembre de 2019.

Philipsen, Kristian (2011), "Entrepreneurship as organizing-A literature study of entrepreneurship", artículo presentado en la conferencia de verano DRUID, 9-11 de junio, Bornholm, <https://bit.ly/3ygwxaN>, 10 de diciembre de 2021.

Quintos, Karen (2019), "Here are four ways to accelerate women entrepreneurs", Ginebra, World Economic Forum, <https://bit.ly/3EfuaXM>, 12 de enero de 2020.

Ruiz Navarro, José; Camelo Ordaz, Carmen, y Coduras Martínez, Alicia (2012), "Mujer y desafío emprendedor en España. Características y determinantes", Economía Industrial, núm. 383, Madrid, Ministerio de Industria, Energía y Turismo, pp. 13-22, <https://bit.ly/3np6OrF>, 4 de octubre de 2019.

Sánchez-Torné, Isadora; Pérez-Suárez, Macarena; García-Río, Esther y Baena-Luna, Pedro (2021), “¿Cómo influye el género en la Intención Emprendedora? Un análisis del estudiantado universitario", Revista de Investigación Educativa, 39 (2), Murcia, Universidad de Murcia, pp. 427-444, doi: https://doi.org/10.6018/ rie. 428451

Shapero, Albert y Sokol, Lisa (1982), "The Social Dimensions of Entrepreneurship", University of Illiniois, Encyclopedia of entrepreneurship, Champaign, The Academy for Entrepreneurial Leadership, pp. 72-90, <https://bit.ly/3IUjSVU>, 1 de septiembre de 2019. 
Sharma, Niharika (2020), "This is how India can tackle unemployment and empower women", Ginebra, World Economic Forum, <https://bit.ly/3G2NOHe>, 16 de julio de 2020 .

Tarapuez Chamorro, Edwin; García González, María Dolly y Castellano, Nélida (2015), "Elementos para la construcción de un instrumento que permita medir la intención empresarial en estudiantes universitarios de último semestre", Tendencias, 16 (2), San Juan de Pasto, Universidad de Nariño, pp. 210227, doi: http://dx.doi.org/10.22267/ rtend.151602.28

Tarapuez Chamorro, Edwin; Guzmán, Beatriz y Parra, Ramiro (2018), "Factores que determinan la intención emprendedora en América Latina", Suma de Negocios, 9 (19), Bogotá, Centro de Investigaciones de la Escuela de Negocios, pp. 5667, doi: https://doi.org/10.14349/sumneg/2018.v9.n19.a7

Taylor, Lianne (2017), "How gender stereotypes are still undermining the capability of female entrepreneurs", Ginebra, World Economic Forum, <https://bit. ly/3lamdI2>, 8 de abril de 2018.

The World Bank (2020), Women, business and the law 2020, Washington, D.C., World Bank Group, <https://bit.ly/3EblscP>, 1 de junio 2020.

The World Bank (2019), "Women, business and the law 2019: a decade of reform", Washington, DC, World Bank Group, <https://bit.ly/3xlcVfo>, 1 de junio de 2020 .

The World Bank (s.f.), "Female Entrepreneurship Resource Point-Introduction and Module 1: Why Gender Matters", Washington, D.C., World Bank Group, <https://bit.ly/2XF3eRm>, 16 de diciembre de 2019.

Urbano, David y Toledano, Nuria (2008), Invitación al emprendimiento, Barcelona, Editorial UOC.
Recibido: 14 de octubre de 2021. Aceptado: 16 de noviembre de 2021. Publicado: 6 de enero de 2022.

\section{Flor Brown-Grossman}

Es doctora en Ciencias Económicas por la Universidad Autónoma Metropolitana. Actualmente es docente investigadora en la Facultad de Contaduría y Administración de la Universidad Autónoma de Querétaro. Es miembro del Sistema Nacional de Investigaciones, nivel III. Sus líneas de investigación son organización industrial, cambio técnico y productividad; economía de género, condiciones y transiciones laborales. Entre sus más recientes publicaciones destacan, como coautora: "Innovative Factors Affecting the Diffusion of the New Nanotechnology Paradigm, 1983-2013", Seoul Journal of Economics, 34 (3), Seúl, Seoul National University-Institute of Economic Research, pp. 329-364 (2021); "Conditional factors Pushing catch-up across developed and emerging countries in the nanotechnology sector, 2000-2010", Economics of Innovation and New Technology, 29 (1), Londres, Taylor \& Francis, pp. 1-19 (2020), y "La política gubernamental en la industria aeronáutica: un análisis comparativo de México, Brasil y España”, Perfiles Latinoamericanos, 28 (55), Ciudad de México, Facultad Latinoamericana de Ciencias Sociales, Sede Académica de México, pp. 253-274 (2020).

\section{Michael Demmler}

Es doctor en Ciencias Económicas por la Universidad de Bayreuth. Actualmente es docente investigador en la Facultad de Contaduría y Administración de la Universidad Autónoma de Querétaro (UAQ). Además, es coordinador del Doctorado en Ciencias Económico Administrativas de la UAQ. Es miembro del Sistema Nacional de Investigadores, nivel I. Sus líneas de investigación son las áreas de finanzas conductuales, burbujas financieras y cultura organizacional. Entre sus más recientes publicaciones se encuentran, como coautor: "Bitcoin and the Sou- 
th Sea Company: A Comparative Analysis", Revista Finanzas y Política Económica, 13 (1), Bogotá, Universidad Católica de Colombia, pp. 197-224 (2021); "Influencia del sexo de los directores generales en el perfil de riesgo, desempeño financiero y la estructura de capital de la empresa: una revisión de la literatura", Revista de Economía, 38 (96), Mérida, Universidad Autónoma de Yucatán, pp. 47-70 (2021), y "Qualitative Análisis of the Current Situation and Future Prospects of the Housing Market in Querétaro", Revista RAITES, 6 (12), Purísima del Rincón, Instituto Tecnológico Superior de Purísima del Rincón, pp. 10-27 (2020).

\section{Selene María López Muñoz}

Es maestra en Ciencias Económicas Administrativas por la Universidad Autónoma de Querétaro. Sus líneas de investigación son en las áreas de economía, administración y género. 